\title{
In-Ear Accelerometer-Based Sensor for Gait Classification
}

\author{
Clara Piris, Lea Gärtner, Miguel A. González, Jérôme Noailly, Fabian Stöcker, Martin Schönfelder, \\ Tim Adams and Simone Tassani
}

\begin{abstract}
For several years, the detection of gait has been popularly implemented using wearable sensors, especially in the sports and medical areas. They are unobtrusive devices which allow to monitor individuals without the need of any ambulatory technology. Despite the fact, the optimal location of the sensor remains uncertain and dependent on the type of measurement. Ear-worn sensors provide a tactical position, robust against movement, that might be significant for gait classification. The purpose of this paper is to demonstrate the accuracy and reliability of in-ear accelerometer sensor to perform gait classification, between the activities walking and running. The data was collected from fourteen participants using an in-ear sensor called ' $C$ osinuss ${ }^{\circ}$ One', which contains a three-dimensional accelerometer sensor. The main characteristics between these two activities were detected using 17 time domain features, as for instance the maximums and standard deviations of the 3-axes, and 3 different window sizes were evaluated: $3.75 \mathrm{~s}, 2 \mathrm{~s}$ and $1 \mathrm{~s}$. Support vector machine (SVM) and $k$-nearest neighbors (KNN) classifiers were implemented and later compared. The total number of features was reduced to 6 for SVM and 12 for KNN preserving the same results. An accuracy over $99 \%$ for both classifiers was achieved, outperforming most of the previous studies.
\end{abstract}

Index Terms - accelerometer sensor, gait, human activity recognition, in-ear sensor, k-nearest neighbors, support vector machines, wearable sensors.

\section{INTRODUCTION}

$\mathrm{O}$ VER the last few years, medical and sport researchers have focused on human activity recognition (HAR), which enables the identification of daily life activities [1], [2]. This area is particularly useful in preventive fitness and medicine, due to its potential for analysis of sports performance and diagnosis of medical conditions [1], [3], [4]. This technology is nowadays still in an early research state, because of the large variety of activities, human characteristics and capture methods [2]. The detection of activities, such as walking or running, is effective in terms of making it possible to identify athletes' performance in order to improve it, or in medicine, providing feedback to the caregiver (e.g. a doctor) about the behavior of the patient. A gait classification of these two basic human

This work was partially funded by the MICINN (HOLOA-DPI2016-80283C2-1-R, RYC-2015-18888), MDM-2015-0502, AEI/FEDER and DTIC-UPF.

Clara Piris was with University Pompeu Fabra Barcelona, Spain and is now with Cosinuss GmbH in Munich, Germany (e-mail: clara.piris17@gmail.com).

Lea Gärtner is with the Chair of Exercise Biology, Department of Sports and Health Sciences of the Technical University of Munich (e-mail: leanadja.gaertner@yahoo.de).

Miguel A. González is with BCN MedTech, DTIC, University Pompeu Fabra, Barcelona, Spain and ICREA, Barcelona, Spain (e-mail: ma.gonzalez@upf.edu). activities is the foundation towards the prevention of injuries and monitoring of health conditions [3], [5]-[8].

The gold standard for gait analysis is stereophotogrammetry. Analysis of gait is carried out with multiple synchronized cameras and optical markers placed at reference points of the body [9]. Therefore, gait features from video sequences can be extracted [7], [8], [10], [11]. This system achieves high accuracy, but it is complex and expensive and needs specialized laboratories [3], [12], [13].

As an alternative, it has become popular to perform gait detection with wearable sensors (WS) using tri-axial accelerometers [1], [10], [14], [15]. WS can give accurate and reliable results about people's activities and behavior [2], [3], [7], [8], [16], [17]. The wearables are unobtrusive devices, which have a comfortable portability. Their form and weight permit users to acquire more autonomy. Moreover, they allow monitoring individuals during large periods of time and without space constraints, i.e. a specialized laboratory. Thus, they facilitate patients, which suffered from a heart attack or Parkinson's disease, to recover from home [18]-[20]. Additionally, their widespread adoption has broken down the cost-prohibitive barriers that existed in the past [4], [5], [14], [15], [21], [22].

Regarding WS placement a wide range of viable locations exist, and the most adequate one varies together with the measurement. Occasionally multiple sensors are used simultaneously, even though placing too many sensors can be heavy, uncomfortable and lead to errors [2], [5], [17]. According to the literature the most common positions for activity recognition are waist, arm, leg, chest, wrist, hip or ankle [2], [5], [16], [17].

Nonetheless, ear-worn sensors are emerging as a promising future in activity recognition, for instance in detecting gait patterns [10], [23]-[26]. During motion the head is the steadiest part of the body and the least affected by the movement. The head stays steadier than the trunk [10], [27]. Atallah et al. in [10] noted that, for example in walking, the direction of the head's movement is more representative of the body's movement than the trunk. For this reason, the detected accelerations in the head are notably attenuated and more

Jérôme Noailly is with BCN MedTech, DTIC, University Pompeu Fabra, Barcelona, Spain (e-mail: jerome.noally@upf.edu)

Fabian Stöcker is with the Teaching and Educational Laboratory, Department of Sport and Health Sciences of the Technical University of Munich (e-mail: fabian.stoecker@tum.de).

Martin Schönfelder is with the Chair of Exercise Biology, Department of Sports and Health Sciences of the Technical University of Munich (e-mail: martin.schoenfelder@tum.de).

Tim Adams is with Cosinuss GmbH in Munich, Germany (e-mail: tim.adams@cosinuss.com).

Simone Tassani is with BCN MedTech, DTIC, University Pompeu Fabra, Barcelona, Spain (e-mail: simone.tassani@upf.edu). 
regular, providing better results and a more realistic human gait analysis [4], [8], [10], [27].

Inertial sensors, such as accelerometers, are an important group inside WS and have been of great importance in monitoring of human activity. Accelerometers calculate the sum of inertial linear and gravitational accelerations along its sensitive axis [7], [28]. In the last years, single-, dual- and triaxis accelerometers have allowed a recognition with high accuracy and low cost of daily life activities [5], [8], [14], [28][31].

The classification of gait can be accomplished implementing machine learning techniques to the accelerometer signal. Multiple data mining and pattern recognition techniques are used to reduce multi-dimensional data and identify patterns [5], [32], [33]. The support vector machine (SVM) and k-nearest neighbors (KNN) classifiers are popularly used for activity detection [1], [2], [10], [14], [25], [33].

In previous works multiple sensors around the body have been repeatedly used in activity recognition, even though a single and light sensor has been proven to be more comfortable and easily accepted by the users [8], [16], [24]. Besides, most of the groundwork makes use of inertial accelerometer sensors, but only a minority focuses on ear-worn accelerometer sensors. Part of these studies aim to detect gait analysis and gait impairment using ear-worn sensors [8], [10], [16], [24]-[26], [30], [34]. The others concentrate in activity recognition, but they approach different methodologies. Atallah et. al. in [5] compare different sensor positions and validates the ear sensor. Pansiot et al. in [35] fusion an ear sensor together with a vision sensor and Atallah et.al in [36] combine an ear sensor with a wireless ambient sensor. Jarchi et al. in [37] detect transitions and classify activities using a singular spectrum analysis. Only Loh et al. [1] make a classification of different activities employing a head-worn accelerometer, but combining it with a barometer pressure sensor and GPS. Alternatively, this study develops a gait classification, of the activities walking and running, using only a single $3 \mathrm{D}$ commercial accelerometer inear sensor, called Cosinuss ${ }^{\circ}$ One.

\section{METHODS}

\section{A. In-Ear Sensor: Cosinuss ${ }^{\circ}$ One}

The wearable used for this study is a 3D accelerometer in-ear sensor called Cosinuss ${ }^{\circ}$ One (shown in Fig. 1). The device, developed by the German start-up Cosinuss $\mathrm{GmbH}$, is used as a professional fitness tracker. The sensor has a size of $45 \times 38$ $\mathrm{x} 18 \mathrm{~mm}$ and a weight of 6.5 grams. The sampling rate is fixed to $100 \mathrm{~Hz}$. ${ }^{\circ}$ One consists of a silicon sensor head with a thermoresistant sensor and an optical pulse oximeter unit, which are in contact with the skin inside the ear canal. The device is made up with 3 different sensors: an optical heart rate sensor (LED and photodiode), a resistance temperature sensor and a 3D-axis accelerometer. Accordingly, it measures body temperature, heart rate, heart rate variability and accelerometer data. The sensor head is available in three sizes $(\mathrm{S}, \mathrm{M}, \mathrm{L})$, hence the device can be adapted to the different types of anatomical properties between children, adults and elderly. It is possible to wear for long periods of time, since it is stable and robust against movement. The wearable is connected via Bluetooth
Low Energy with a smartphone application developed by the company. In the app it is possible to follow measurements and receive feedback. Afterwards, the data is sent to the cosinuss ${ }^{\circ}$ server where a full analysis is performed [38].

An accelerometer computes the sum of inertial linear and gravitational accelerations along its axes $(x, y$ and $z)$, which represent the 3 different directions during movement. For this device, $y$-axis corresponds to the vertical direction (positive down), $\mathrm{x}$-axis to the anterior-posterior direction (positive back) and $\mathrm{z}$-axis the medio-lateral direction (positive lateral) as shown in Fig. 2. The accelerometer measures proper acceleration whereby the measurements include effects of gravity [7], [39].

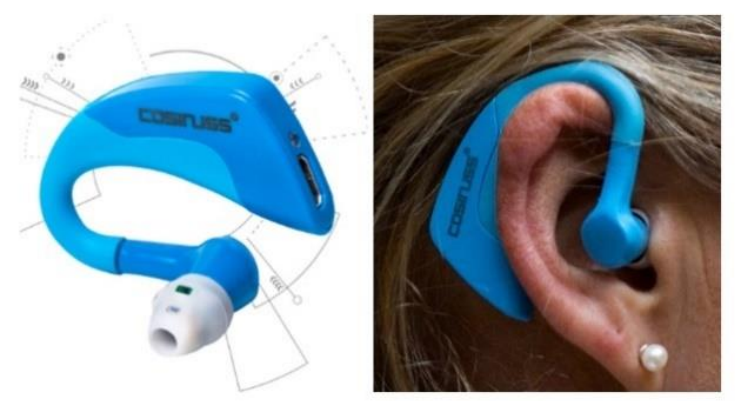

Fig 1. Wearable sensor "Cosinuss ${ }^{\circ}$ One".

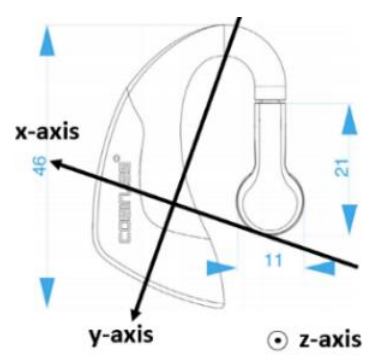

Fig 2. Acceleration directions for Cosinuss $^{\circ}$ One.

\section{B. Subjects and Experimental Procedure}

A total of 14 subjects participated in the experiment. The participants agreed and signed a declaration of consent approved by the Department of Sports and Heath Sciences at Technical University of Munich (TUM). The requirements included healthy subjects between 18 and 50 years old, with no illness, chronical diseases, injures or spine operation in the last 6 months. It was asked not to ingest alcohol or caffeine 24 hours before the test [40], [41]. The tests took place in the "Prevention and Performance Lab" of TUM. The lab conditions were 22 degrees with an air humidity of 45 percent.

The experiment consisted in 3 parts: pre-test, main-test and post-test. The participants executed the 3 tests on the same day, with a duration of approximately 1 hour. The measurement instruments in the pre-test were a $\mathrm{h} / \mathrm{p}$ cosmos pulsar treadmill and a Sony camera. In main-test and post-test the wearable sensors, $\operatorname{Cosinuss}^{\circ}$ One, was used to collect the data. 
Pre-test. This phase allowed to determine the transition speed of the participants. Each subject started walking on the treadmill with a speed of $4 \mathrm{~km} / \mathrm{h}$. Every minute the speed was increased $0.5 \mathrm{~km} / \mathrm{h}$, until the subject felt more comfortable running than walking. The same procedure was done backwards, from running to walking, with an initial speed of 9 $\mathrm{km} / \mathrm{h}$. In order not to bias the results, the speed values were hidden from the subjects.

Main-test. This phase evaluated walking and running at the transition speed previously obtained for each subject. The participants started walking for 2 minutes. When the investigator was giving a signal, they had to start running for 2 more minutes. The shift of activities was executed 3 times, leaving a recording of 12 minutes.

Post-test. This phase evaluated walking under the subject's transition speed and running over their transition speed. After 5 minutes break, the subjects walked at 3,4 and $5 \mathrm{~km} / \mathrm{h}$ for 2 minutes each. Accordingly, they ran at 8,9 and $10 \mathrm{~km} / \mathrm{h}$ for 2 minutes each.

At the end of the experiment, the participants filled out a questionnaire which helped to extract information about the participants (sex, weight, height and age), their experience with other wearables (if they used before and if so, which ones) and their experience with the sensor (if they like it and if they would use it again). This information was important to contextualize the market and better understand the results.

\section{Data Management}

To access the data, it was necessary to connect the sensor via Bluetooth with the smartphone application. Afterwards, the raw data was ready to be downloaded from the internal server and edited. In the first place, the physical information of the participants, obtained in the questionnaire, was added in each file respectively. Later, the samples were labeled differentiating the phases where the subject walked, ran and the transition between the two activities. Walking was labeled as ' 0 ', running as ' 1 ' and the transition as '99'. Subsequently, the transition class, which last approximately 5 to 10 seconds, was eliminated as Benson et al. [33] obtaining a binary classification.

\section{Windows segmentation}

The data was segmented into smaller segments, called windows, which should contain minimum one gait cycle [33]. The ideal window size is an important parameter for a fast and accurate classification. Having small windows can improve the performance, but the computational cost is higher since the algorithm is triggered often. Long windows can include more than one activity and do not always increase the accuracy [2], [3], [33]. Atallah et al. [42] noted that for activities with repeating events such as walking on a treadmill, a window smaller than 1 minute is enough.

Fixed-size sliding window (FSW), in which all the windows have the same fix length, is the most used in gait analysis detection [2], [33]. Overlapping means that a percentage of data from one window, will be found in the following one, smoothening the transition. This improve the capture of relevant information. It is common to use 50\% overlapping [1][3], [33], [43]. FSW with overlap is commonly used in medical research such as patient monitoring [43].
In this paper fixed size sliding windows of $3.75 \mathrm{~s}, 2 \mathrm{~s}$ and $1 \mathrm{~s}$ were chosen to observe how the classifier respond to larger windows (such as $3.75 \mathrm{~s}$ which include 8 run cycles) and smaller ones (such as $1 \mathrm{~s}$ which include 2 run cycles). Also, an overlap of $50 \%$ was used for all the window sizes.

\section{E. Feature extraction}

The next step consisted in extracting information which describe the gait cycle and help to compare two given windows [2], [3]. This step is called, feature extraction.

The magnitude of the accelerometer, or vector length, was calculated. This vector is a representation of the 3-axes and it is relevant for gait detection, since it is less sensible to the effects of sensor orientation [2].

$$
\text { proper acceleration magnitude }=\sqrt{a_{x}{ }^{2}+a_{y}{ }^{2}+a_{z}{ }^{2}}
$$

where $a_{x}, a_{y}, a_{z}$ are the accelerometer axis $x, y$ and $z$.

For each window a total of 17 features were calculated. The main features used for daily life activity recognition with accelerometer data, as defined in the previous works [1]-[3], [5], [33], [42]-[44], were selected. The chosen features belong to the time domain group, due to their low computational cost and high discriminatory ability [2]. To the list of features presented in the literature cross-correlation was added. With this feature we tried to measure the similarity between two axes. Table I shows a list of all the features extracted for this paper.

TABLE I

\section{TIME DOMAIN FEATURES}

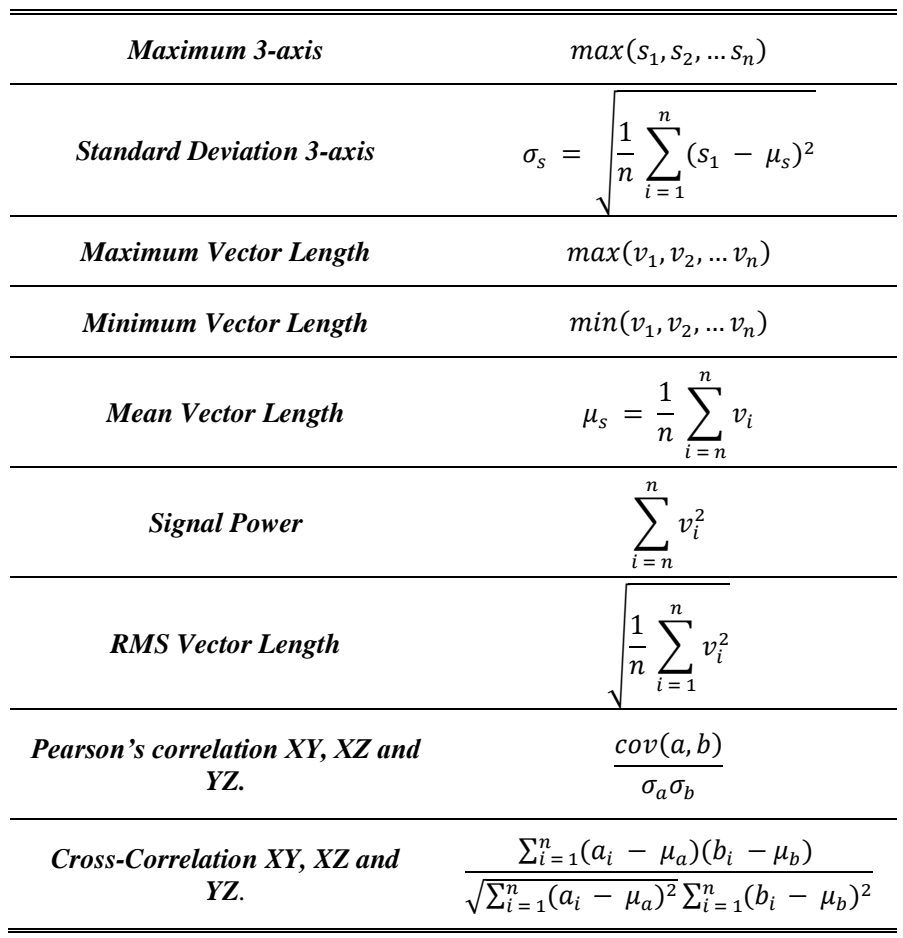

List of implemented features [2], [3], [43] 


\section{F. Feature selection}

After calculating the features, their importance on the prediction of the classification was calculated. The so-called step, feature selection, chooses the most appropriate ones. Some features contain irrelevant information that affect the accuracy adding noise to the signal [3], [5], [42].

Feature selection was carried out with recursive feature elimination with cross-validated selection of the best number of features (RFECV). The initial set of features is recursively reduced estimating the least important features, until a desired number of features is reached. The importance of the features is obtained from an external estimator that assigns weights to features (e.g. the coefficients of a linear model or through a features' importance attribute). A ranking of features is returned, where 1 coincides with the best permutation. A minimum of 4 features was asked for the classification. KNN does not provide a prediction of coefficients or the features' importance (see "Classifier implementation"), therefore a Linear Regression model was used to calculate the coefficients and approach the ranking of features.

\section{G. Classifier implementation}

Afterwards, two typical algorithms in pattern recognition were implemented: support vector machine (SVM) and $k$ nearest neighbor (KNN). The groundwork shows that these 2 classifiers are the most used for activity recognition, confirmed by Janidarmian et al. [2], who compared 293 classifiers in the study.

The previous 17 features were used in the evaluation. For both classifiers, a scaling of data was performed. The models were tested with random split and stratified $k$-fold crossvalidation. The splitting proportion in percent between test and train were: $[34,66],[50,50],[60,40]$ and [80,20]. For crossvalidation 10-fold and 5-fold were used. For SVM three types of kernel were evaluated: linear, polynomial and radial basis function $(\mathrm{RBF})$ kernel. The three models shared a regularization parameter and a gamma parameter.

\section{Support Vector Machine Optimization}

Not always a perfect margin between classes can be found, hence a soft-margin SVM allow some of the training data points to be misclassified. Introducing slack variables, a trade-off between margin and training error can be perform. The parameter that regularizes this exchange is $C$, also called as regularization parameter. For low values of $C$, the misclassified data points have low influence on the margin and for high values these data points contribute strongly. Altering this value changes the decision boundary and the classification of the points [45], [46].

The gamma parameter $(\gamma)$ determines the range (or spread) of the points which have more impact. High value of $\gamma$ correspond to a small radius and therefore small and limited distance. Low values equal to large distances and many points are considered [46], [47].

$C$ and $\gamma$ received the values of 1 and 0.001 , respectively. The values were chosen following the SVM algorithm used in WEKA (University of Waikato, Hamilton, New Zealand). Although, for the polynomial kernel, $\gamma$ was equal to 'scale', the predefined value. This kernel has a third parameter called degree, here set to 3 .

\section{K-Nearest Neighbor Optimization}

For KNN two models were tested: uniform and distance. The parameters used for both were: 1 neighbor, algorithm=auto and $\mathrm{p}=2$. The parameter $p$ defines the calculation of the distance between points as standard Euclidean distance. The parameter 'auto' selects the most appropriate algorithm based on the values passed to the fit method. The possible algorithms are: BallTree, KDTree and brute force.

\section{H. Evaluation of Performance}

The main method for evaluation performance was accuracy, defined as the ratio of samples that are correctly predicted. However, sometimes it does not provide with enough information, thus the confusion matrix, precision, recall, $f$ measure and Matthews Correlation Coefficient (MCC) were also calculated.

\section{RESULTS}

\section{A. Subjects: Questionnaire Results}

Table 2 shows the average and standard deviation of the physical characteristics of the participants, among them 9 women and 5 men.

\section{TABLE II}

PHYSICAL CHARACTERISTICS

\begin{tabular}{cc}
\hline \hline Age & $24 \pm 2.23$ \\
Height & $173.00 \pm 8.42 \mathrm{~cm}$ \\
Weight & $66.00 \pm 12.48 \mathrm{~kg}$ \\
\hline \hline
\end{tabular}

VALUES $($ MEAN \pm SD)

A total of 5 participants $(35.71 \%)$ had previously used a wearable sensor for training control. All of them worn a fitness watch. Just one of the participants had problems fitting the sensor at the start. Cosinuss ${ }^{\circ}$ One received a rating of 1.75 on a scale of 1 (very good) to 6 (unsatisfactory). The lowest grade received was equal to 3 .

\section{B. Data management}

Walking and running profiles are shown respectively in Figure 3 (10 cycles) and Figure 4 (11 cycles). In average, the gait cycles, calculated from peak to peak, last about $0.5 \mathrm{~s}$ for walking and $0.4 \mathrm{~s}$ for running. Generally running cycles are more regular, symmetric and short than walking cycles. However, the main difference is the flight phase, which occurs during running when none of the feet touches the ground.

\section{Window segmentation and feature selection}

The study achieved in any combination a classification accuracy superior to $99 \%$ using both SVM and KNN classifiers, different number of features (17, 12 and 6) and different window sizes $(3.75 \mathrm{~s}, 2 \mathrm{~s}$ and $1 \mathrm{~s})$. Therefore, the classifier can be identified as robust and reliable. In Table 3 a summary of all the results is shown.

The feature selection procedure allowed to reduce the initial number of 17 features. For the SVM classifier, it could be reduced to 9 features (in $3.75 \mathrm{~s}$ windowing), 6 features (in $2 \mathrm{~s}$ windowing) and 7 features (in $1 \mathrm{~s}$ windowing). On the other hand, for the KNN the optimal minimum number was 12 features for the different segmentations. 

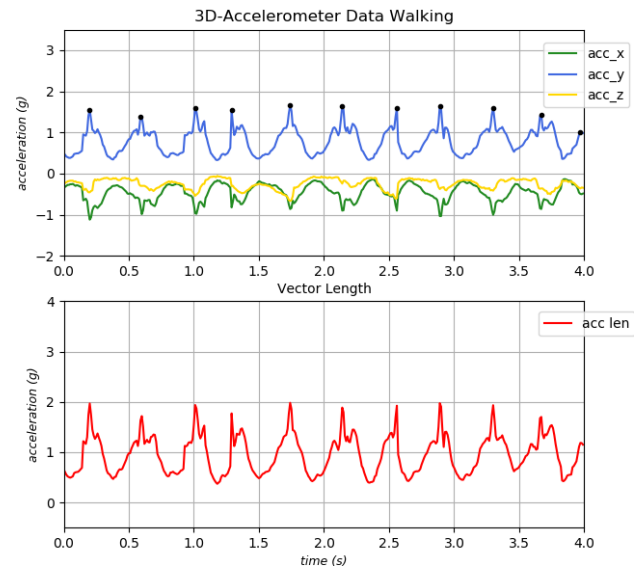

Fig 3. Upper graph shows the 3D accelerometer data of a subject while walking. The green signal corresponds to $\mathrm{x}$-axis accelerometer data, the blue to the $y$-axis and the yellow in the z-axis. Peaks are marked with black points. In the lower graph, the vector length of the same segment is represented in
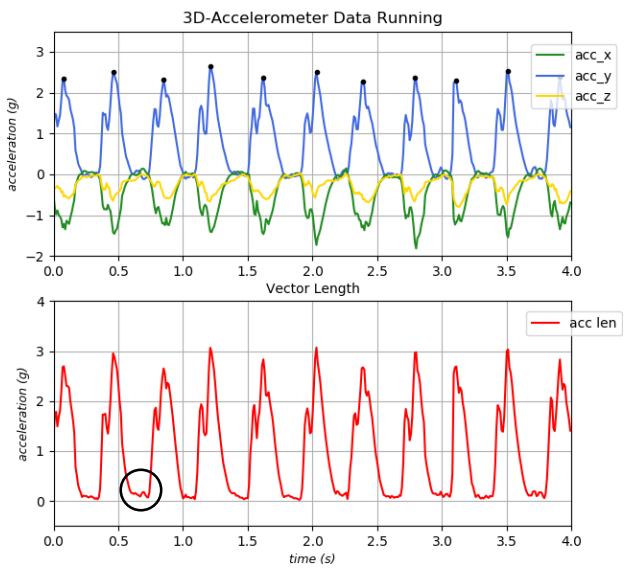

Fig 4. The graph shows the 3D accelerometer data and vector length of a subject while running. Peaks are marked with black points. Flight phase is detected between cycles, where the vector length's acceleration varies around zero. red.

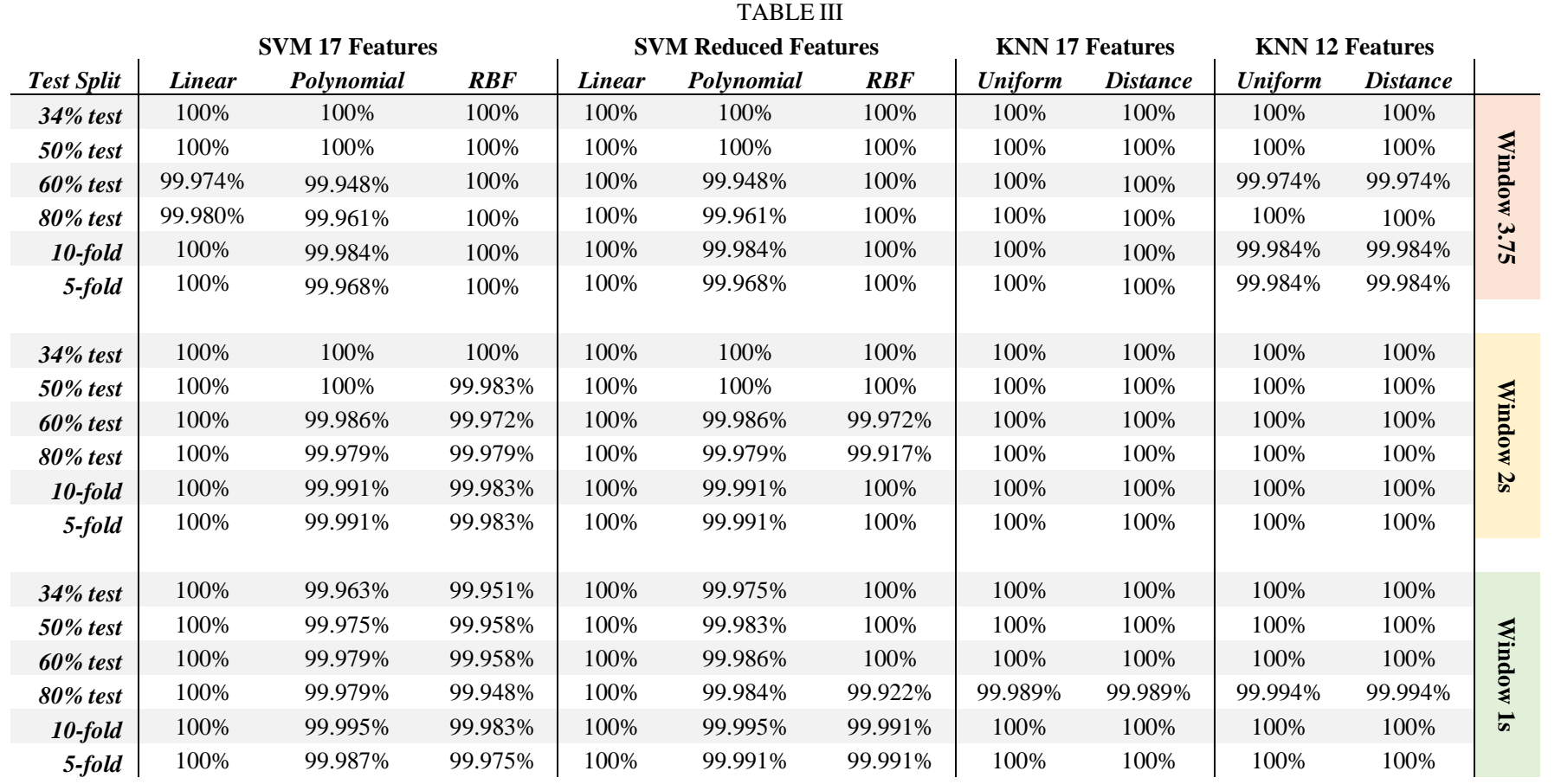

Summary of accuracy results. The first column corresponds to the way the data was split. The second and third columns are the results of the support vector machine (SVM) for the linear, polynomial and radial basis function (RBF) kernel. Using 17 and a reduced number $(9,6$ and 7$)$ of features. The two followings ones are the results of the $k$-nearest neighbors $(\mathrm{KNN})$ using 17 and 12 features.

It coincides for both classifiers that the results when the number of features is decreased are significantly better than using all the features and, also that the $3.75 \mathrm{~s}$ segmentation achieves the worse results.

Observing in detail the SVM with 8 features (in 2s and 1s windows), it is noticeable that most of the outcomes in the polynomial kernel are higher for the $2 \mathrm{~s}$ segmentation than the 1s one. Diversely, in the RBF kernel it depends on the way the data is split. In train-test-split, the $1 \mathrm{~s}$ segmentation obtains better results, but when the cross-validation is calculated, the $2 \mathrm{~s}$ window obtain higher results (reaching a 100\% accuracy). The KNN with 12 features achieves almost equal results for both windows. The only difference is found specifically when an $80 \%$ of the data is designated to test. In the $1 \mathrm{~s}$ window, the accuracy decreases to a $99.994 \%$, value which is still considered very good. The confusion matrix in Table 4 shows that this accuracy corresponds to just 1 misclassification in the running data (19329). The ranking of features for the SVM with $2 \mathrm{~s}$ windows and the KNN with $1 \mathrm{~s}$ windows is graphically illustrated in Figure 5.

TABLE IV

\begin{tabular}{ccccccc}
\multicolumn{2}{c}{ CONFUSION MATRIX } & PRECISION & RECALL & F1SCORE & MCC \\
\hline \hline & walk & run & & & & 0.99 \\
\hline walk & 9691 & 0 & 1.00 & 1.00 & 1.00 & \\
\hline run & 1 & 9637 & 1.00 & 1.00 & 1.00 & \\
\hline \hline
\end{tabular}

Evaluation of performance for the train-test split with $80 \%$ of the data assigned to test and using the KNN classifier with 12 features. Performed metrics used: confusion matrix, precision, recall, f-measure and MCC. 


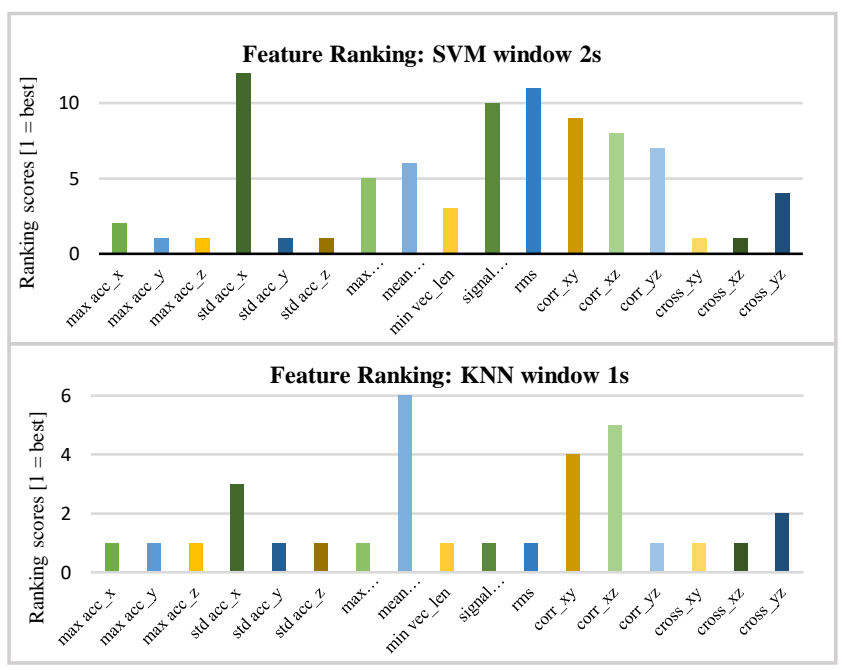

Fig 5. Ranking of features for the 2 selected combinations: support vector machine (SVM) with $2 \mathrm{~s}$ windowing and k-nearest neighbor (KNN) with $1 \mathrm{~s}$ windowing. The best features have a score equal to 1 . The features are maximums and standard deviations of the 3 -axes, maximum, minimum and mean of the vector length, signal power, root mean square (rms), Pearson's correlation between axes $x y, x z, y z$ and cross-correlation of axes $x y, x z, y z$.

\section{CONCLUSIONS}

The classification results achieve for any configuration an accuracy over 99\%. Although, the best combination is considered the one which reach the best classifier performance with the smallest number of features. As a result, the composition of window size $2 \mathrm{~s}$ with 6 features is chosen for SVM and window size $1 \mathrm{~s}$ with 12 features for KNN. The window size 3.75s appears to include too much information, what increases the misclassification. It must be highlighted that the best 6 features in the SVM are also found in the KNN classification. Those features are the maximums of the axes $y$ and $z$, the standards deviation of the axes $y$ and $z$, and the crosscorrelation of the axes $x-y$ and $x-z$. Essentially what those features do is climax the dissimilarities of the two activities, mainly measuring the primary differences between these axes' signals, such as maximums and standards deviations. Possibly, these features would decrease performance if the sensor would be positioned somewhere less stable and more affected by the movement, since more noise would be added. It is noteworthy that the classification is carried out exclusively with timedomain features to allow a low computation cost. The outstanding results confirm the ear as a strategic point for sensor positioning if gait classification is aimed.

Considering robustness against overfitting and reliability, the cross-validation method is preferred over the train-test-split. Further consideration is the computational load, hence 5-fold cross-validation is chosen instead of 10-fold, since they basically obtained the same accuracy results.

The activities walking and running are very similar and not always easily separable, nevertheless in this study the classifiers that operate more effectively were those which use a linear hyperplane, such as SVM with linear kernel and KNN. Also, a chance of overfitting is reduced by working with linear borders.

Positioning the sensor in the ear offers more stable signals than other body locations ergo better results, proving the efficiency and effectiveness of the sensor location. The high performance of the classifiers demonstrates that an in-ear sensor, which nowadays are still rarely used for gait classification, can reach results at the level of measurements obtained around the body's center of gravity and that the accelerations acquired in the head permit a reliable and wellgrounded gait detection. It is also proven that the gait classification can be performed with a high accuracy using just one single sensor in the ear, avoiding the discomfort of wearing multiple sensors around the body.

In comparison with Loh et al. [1], the single study which classifies between activities, our classification reached values of $f$-measure equal to $100 \%$ for both activities, without using gyroscope and barometric pressure sensors' measurements.

Nonetheless, as with most studies, these results must be interpreted with caution and a few limitations should be borne in mind. Even though the obtained number of samples is large, the number of subjects is low and unbalanced (5 men and 9 women). In addition, all the participants are healthy subjects and belong to the same age group around 24 years old. Another category of subject, such as elderly, obese subjects or patients with disabilities, might influence the results. The addition of these categories could allow to evaluate the effect of age, body mass index (BMI) or injury in the classification. A further extension of this study could be to analyze walking and running movements, identifying gait phases such as swing and stance phase or step length for healthy subjects. Subsequently, the study could proceed with the classification of pathological movements, such as gait abnormalities.

In conclusion, this study achieved an accuracy higher than most of the previous literature, using a small number of features and with a single ear worn sensor.

\section{ACKNOWLEDGMENT}

The authors would like to thank all the students who participated in this study.

\section{REFERENCES}

[1] D. Loh, T. J. Lee, S. Zihajehzadeh, R. Hoskinson, and E. J. Park, "Fitness activity classification by using multiclass support vector machines on head-worn sensors," Proc. Annu. Int. Conf. IEEE Eng. Med. Biol. Soc. EMBS, vol. 2015-Novem, pp. 502-505, 2015.

[2] M. Janidarmian, A. R. Fekr, K. Radecka, and Z. Zilic, "A comprehensive analysis on wearable acceleration sensors in human activity recognition," Sensors (Switzerland), vol. 17, no. 3, 2017.

[3] Ó. D. Lara and M. A. Labrador, "A Survey on Human Activity Recognition using Wearable Sensors," IEEE Commun. Surv. Tutorials, vol. 15, no. 3, pp. 63-66, 2013.

[4] J.-H. Park, D.-G. Jang, J. Park, and S.-K. Youm, "Wearable Sensing of In-Ear Pressure for Heart Rate Monitoring with a Piezoelectric Sensor," Sensors, vol. 15, no. 9, pp. 23402-23417, Sep. 2015.

[5] L. Atallah, B. Lo, R. King, and G. Z. Yang, "Sensor placement for activity detection using wearable accelerometers," 2010 Int. Conf. Body Sens. Networks, BSN 2010, pp. 24-29, 2010.

[6] M. Oner, J. A. Pulcifer-Stump, P. Seeling, and T. Kaya, "Towards the run and walk activity classification through step detection - An android application," in 2012 Annual International Conference of the IEEE Engineering in Medicine and Biology Society, 2012, pp. 19801983.

[7] W. Tao, T. Liu, R. Zheng, and H. Feng, "Gait Analysis Using Wearable Sensors," Sensors, vol. 12, no. 2, pp. 2255-2283, Feb. 2012. 
T.-H. Hwang, J. Reh, A. O. Effenberg, and H. Blume, "Real-Time Gait Analysis Using a Single Head-Worn Inertial Measurement Unit," IEEE Trans. Consum. Electron., vol. 64, no. 2, pp. 240-248, May 2018.

[9] A. Cappozzo, U. Della Croce, A. Leardini, and L. Chiari, "Human movement analysis using stereophotogrammetry: Part 1: theoretical background," Gait Posture, vol. 21, no. 2, pp. 186-196, Feb. 2005.

[10] L. Atallah, B. Lo, G. Z. Yang, and O. Aziz, "Detecting walking gait impairment with an ear-worn sensor," IEEE, pp. 175-180, 2009.

[11] G. Guerra-Filho, "Optical motion capture: Theory and implementation," J. Theor. Appl. Informatics, vol. 12, pp. 61--89, 2005.

[12] R. Baker, "Gait analysis methods in rehabilitation," $J$ NeuroEngineering Rehabil, vol. 3, no. 1, p. 4, 2006.

[13] P. Eichelberger et al., "Analysis of accuracy in optical motion capture - A protocol for laboratory setup evaluation," J. Biomech., vol. 49, no. 10, pp. 2085-2088, Jul. 2016.

[14] C. A. Clermont, L. C. Benson, S. T. Osis, D. Kobsar, and R. Ferber, "Running patterns for male and female competitive and recreational runners based on accelerometer data," J. Sports Sci., vol. 37, no. 2, pp. 204-211, Jan. 2019.

[15] D. Quintero, D. J. Lambert, D. J. Villarreal, and R. D. Gregg, "RealTime continuous gait phase and speed estimation from a single sensor," 1st Annu. IEEE Conf. Control Technol. Appl. CCTA 2017, vol. 2017-Janua, pp. 847-852, 2017.

[16] D. Jarchi, B. Lo, C. Wong, E. Ieong, D. Nathwani, and G. Z. Yang, "Gait Analysis from a Single Ear-Worn Sensor: Reliability and Clinical Evaluation for Orthopaedic Patients," IEEE Trans. Neural Syst. Rehabil. Eng., vol. 24, no. 8, pp. 882-892, 2016.

[17] F. Attal, S. Mohammed, M. Dedabrishvili, F. Chamroukhi, L. Oukhellou, and Y. Amirat, "Physical human activity recognition using wearable sensors," Sensors, vol. 15, no. 12, pp. 31314-31338, 2015.

[18] S. C. Mukhopadhyay, "Wearable Sensors for Human Activity Monitoring: A Review," IEEE Sens. J., vol. 15, no. 3, pp. 1321-1330, Mar. 2015.

[19] S. V. Perumal and R. Sankar, "Gait and tremor assessment for patients with Parkinson's disease using wearable sensors," ICT Express, vol. 2, no. 4, pp. 168-174, Dec. 2016.

[20] A. R. Anwary, H. Yu, and M. Vassallo, "An Automatic gait feature extraction method for identifying gait asymmetry using wearable sensors," Sensors (Switzerland), vol. 18, no. 2, Feb. 2018.

[21] P. Bonato, "Wearable Sensors / Systems and Their Impact on Biomedical Engineering: An Overview from the Guest Editor," IEEE Eng. Med. Biol. Mag., no. June, 2003.

[22] B. Najafi, T. Khan, and J. Wrobel, "Laboratory in a box: Wearable sensors and its advantages for gait analysis," in Proceedings of the Annual International Conference of the IEEE Engineering in Medicine and Biology Society, EMBS, 2011.

[23] B. Lo, L. Atallah, O. Aziz, M. El Elhew, A. Darzi, and G. Yang, "Real-Time Pervasive Monitoring for Postoperative Care Real-Time Pervasive Monitoring for Postoperative Care," no. May 2014, 2007. D. Jarchi, C. Wong, R. M. Kwasnicki, B. Heller, G. A. Tew, and G. Z. Yang, "Gait parameter estimation from a miniaturized ear-worn sensor using singular spectrum analysis and longest common subsequence," IEEE Trans. Biomed. Eng., vol. 61, no. 4, pp. 1261$1273,2014$.

[25] L. Atallah et al., "Validation of an ear-worn sensor for gait monitoring using a force-plate instrumented treadmill," Gait Posture, vol. 35, no. 4, pp. 674-676, Apr. 2011.

[26] L. Atallah, A. Wiik, B. Lo, J. P. Cobb, A. A. Amis, and G. Z. Yang, "Gait asymmetry detection in older adults using a light ear-worn sensor," Physiol. Meas., vol. 35, no. 5, May 2014.

[27] J. J. Kavanagh, S. Morrison, and R. S. Barrett, "Coordination of head and trunk accelerations during walking," Eur. J. Appl. Physiol., vol. 94, no. 4, pp. 468-475, 2005.

[28] V. Camomilla, E. Bergamini, S. Fantozzi, and G. Vannozzi, "Trends supporting the in-field use of wearable inertial sensors for sport performance evaluation: A systematic review," Sensors (Switzerland), vol. 18, no. 3, 2018.

[29] M. Ermes, J. Pärkkä, J. Mäntyjärvi, and I. Korhonen, "Detection of daily activities and sports with wearable sensors in controlled and uncontrolled conditions," IEEE Trans. Inf. Technol. Biomed., 2008.

[30] L. Li, L. Atallah, B. Lo, and G. Z. Yang, "Feature extraction from ear-worn sensor data for gait analysis," 2014 IEEE-EMBS Int. Conf.
Biomed. Heal. Informatics, BHI 2014, pp. 560-563, 2014.

[31] S. Sprager and M. Juric, "Inertial Sensor-Based Gait Recognition: A Review," Sensors, vol. 15, no. 9, pp. 22089-22127, Sep. 2015.

[32] R. Ferber, S. T. Osis, J. L. Hicks, and S. L. Delp, "Gait biomechanics in the era of data science," J. Biomech., vol. 49, no. 16, pp. 37593761, 2016.

[33] L. C. Benson, C. A. Clermont, S. T. Osis, D. Kobsar, and R. Ferber, "Classifying running speed conditions using a single wearable sensor: Optimal segmentation and feature extraction methods," J. Biomech., vol. 71, pp. 94-99, Apr. 2018.

[34] L. Atallah, O. Aziz, E. Gray, B. Lo, and G.-Z. Yang, "An Ear-Worn Sensor for the Detection of Gait Impairment After Abdominal Surgery," Surg. Innov., vol. 20, May 2012.

[35] J. Pansiot, D. Stoyanov, D. McIlwraith, B. Lo, and G. Yang, Ambient and Wearable Sensor Fusion for Activity Recognition in Healthcare Monitoring Systems, vol. 13. 2007.

[36] L. Atallah, B. Lo, R. Ali, R. King, and G. Yang, "Real-Time Activity Classification Using Ambient and Wearable Sensors," IEEE Trans. Inf. Technol. Biomed., vol. 13, no. 6, pp. 1031-1039, 2009.

[37] D. Jarchi, L. Atallah, and G. Yang, "Transition Detection and Activity Classification from Wearable Sensors Using Singular Spectrum Analysis," in 2012 Ninth International Conference on Wearable and Implantable Body Sensor Networks, 2012, pp. 136141.

[38] Cosinuss GmbH, "Cosinuss In-Ear Sensor,” 2019.

[39] S. Buddies, “Accelerometer Technical Note," pp. 2-7, 2020.

[40] P. Avogadro, A. Dolenec, and A. Belli, "Changes in mechanical work during severe exhausting running," Eur. J. Appl. Physiol., vol. 90, no. 1-2, pp. 165-170, Sep. 2003.

[41] L. D. Wilkin, A. Cheryl, and B. L. Haddock, "Energy expenditure comparison between walking and running in average fitness individuals," Journal of Strength and Conditioning Research. 2012.

[42] L. Atallah, J. J. H. Leong, B. Lo, and G.-Z. Yang, "Energy Expenditure Prediction Using a Miniaturized Ear-Worn Sensor," Med. Sci. Sport. Exerc., vol. 43, no. 7, pp. 1369-1377, Jul. 2011.

[43] S. Bersch, D. Azzi, R. Khusainov, I. Achumba, and J. Ries, "Sensor Data Acquisition and Processing Parameters for Human Activity Classification," Sensors, vol. 14, no. 3, pp. 4239-4270, Mar. 2014.

[44] I. Farkas and E. Doran, "ACTIVITY RECOGNITION FROM ACCELERATION DATA COLLECTED WITH A TRI-AXIAL ACCELEROMETER," ACTA Tech. NAPOCENSIS Electron. Telecommun., vol. 52, no. 2, pp. 38-43, 2011.

[45] C. M. Bishop, Machine Learning and Pattern Recoginiton. 2007.

[46] A. C. Müller and S. Guido, Introduction to machine learning with Python. 2016.

[47] D.-I. M. Dorr, Pattern Recognition Lecture Notes in Computer Science. 2018.

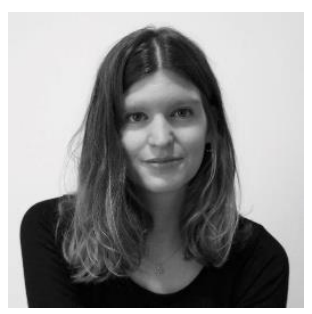

Clara Piris Burgos holds a bachelor in Audiovisual Systems Engineering from the Universitat Pompeu Fabra de Barcelona (UPF). After an international exchange at the Technische Universität München (TUM), she started an internship in the company Cosinuss $\mathrm{GmbH}$ in Munich. She wrote her final degree work in the company with the supervision of the UPF. Her bachelor thesis was awarded as one of the best bachelor projects of her degree.

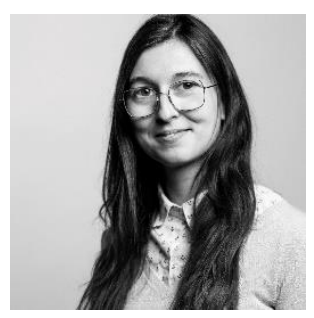

Lea Gärtner holds a bachelor in Scientific Principles of Sports from the Technische Universität München (TUM) and a master in Diagnostics and Training from the same university. One year later she started the MSc in Ergonomics - Human Factors Engineering. She performed her master thesis in the company Cosinuss $\mathrm{GmbH}$. She is currently working as a test engineer in AKKA DSO GmbH. 


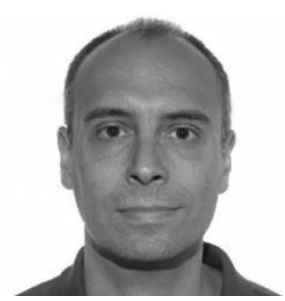

Dr. Miguel A. González Ballester holds a computer science degree from Universitat Jaume I, Spain (1996) and a doctoral degree from the University of Oxford, UK (2000). His doctorate, under supervision of Sir Michael Brady and Prof. Andrew Zisserman, focused on the analysis of brain MRI data for multiple sclerosis and schizophrenia. He was awarded the prestigious Toshiba Research Fellowship and moved to Japan to work for two years as a senior researcher at Toshiba Medical Systems, where he developed novel, patented systems for MRI parallel imaging. In late 2001 he obtained a faculty position at INRIA (Sophia Antipolis, France), where he led research projects on medical image analysis and mathematical modelling. In 2004 he joined the University of Bern (Switzerland), as head of the medical image analysis group, and later became head of the surgical technology division at the Faculty of Medicine. From 2008 until September 2013 he oversaw the Research Department of the company Alma IT Systems in Barcelona (Spain), where he led the development of a new generation of computer tools for diagnosis and surgical planning. In October 2013 he was awarded an ICREA Senior Research Professorship and joined the Department of Information and Communication Technologies at Universitat Pompeu Fabra in Barcelona, where he leads the Barcelona Center for New Medical Technologies (BCN MedTech, 60+ staff).

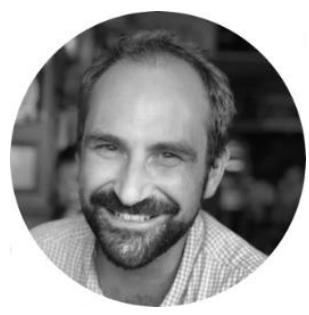

Dr. Jérôme Noailly holds a bachelor in Physical Chemistry and an engineer's degree in Material Engineering. He started his $\mathrm{PhD}$ in late 2002 at the Universitat Politècnica de Catalunya (UPC), where he explored the lumbar spine biomechanics through nonlinear finite element modelling. From 2007 to 2011, Jérôme focused on soft tissue and multiphysics modelling as a Marie Sklodowska-Curie postdoctoral fellow, at the AO Foundation and the Eindhoven University of Technology, and at the Institute for Bioengineering of Catalonia (IBEC). In 2012, he became the principal investigator (PI) of the group of Biomechanics and Mechanobiology at IBEC and started to extend his research to systems biology. He eventually moved to UPF, where he is leading the Biomechanics \& Mechanobiology research area of the BCN MedTech research unit. His team focusses on the multiscale exploration of load-bearing organs, tissues and cellular systems of the human body, from motion analysis to biological regulation.

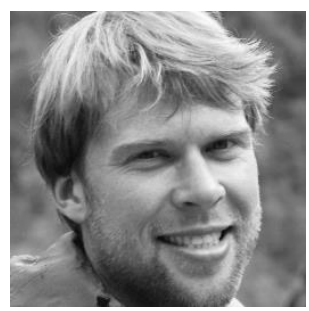

Dr. phil. Fabian Stöcker holds a diploma in sports sciences from the Technische Universität München (TUM). The topic of his diploma's thesis was "Vibration training: Changing of strength and coordination parameters through two different training forms". Afterwards in 2016 he got promoted to philosophy doctor. His topic of dissertation was "Muscle oxygen availability in response to exercise: influence of intensity and duration". From 2011 to 2012 he worked in the GeWoS-Project (Gesund Wohnen mit Stil) from the department of Preventive Pediatrics. From 2012 to 2015 he was part of the scientific staff at the chair for Training Science Diagnostics (Trainingwissenschaftliche Diagnostik). Since 2011 he is part of the Department for Sports and Health Sciences at TUM and head of the teaching and learning laboratory.

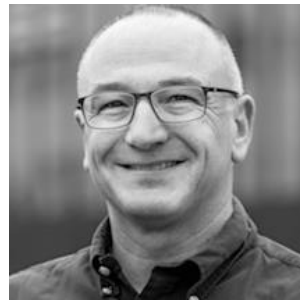

Dr. Martin Schönfelder hold a master's degree (Staatsexamen) in Biology and Sports at the Heidelberg University. In 2000 he started his PhD (magna cum laude) in Molecular Physiology, with the topic "Gene expression analysis in invitro maturated bovine oocytes) at the Technische Universität München (TUM). From 2004 to 2012 he worked in the Lecturer in Sport Physiology at TUM. In 2015 he worked in the Research Professorship Paracelsus Private University in Salzburg, Austria. Afterwards he was head of the laboratory of Neuropathology at TUM. Since 2016 he is a Senior lecturer and head of laboratory of the department of Exercise Biology at TUM.

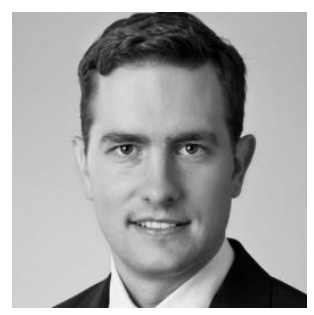

Dr. rer. nat. Tim Adams holds a diploma in Physics from the Technische Universität München (TUM) with the areas of focus magnetism, spintronics and astrophysics. He also has $\mathrm{a} \mathrm{PhD}$ at the chair of strongly correlated electron systems from TUM. His doctorate topic was "Neutron Scattering on the Skyrmion Lattice in Chiral Magnets". From 2004 to 2009 he was a working student at Sysberry $\mathrm{GmbH}$ with the position of software development and ITadministration. From 2008 to 2011 he was honorary project lead and board of management in the European Juggling Convention 2011. Since 2015 he is the leader of the Software team in Cosinuss GmbH.

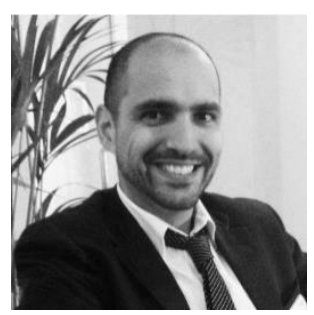

Dr. Simone Tassani holds a Master and $\mathrm{PhD}$ Doctorate from the Alma Mater Studiorum University of Bologna (Italy) in bioengineering and biomechanics with specialization in trabecular bone microstructure, its mechanical behaviour and remodelling in pathologies like osteoarthritis and osteoporosis. He was awarded with a Marie- Curie Intra-European Individual fellowship in 2010 and he moved to Greece where he worked on the biomechanical analysis of bone trabecular structure, through the development of image processing tools for the identification and classification of trabecular bone fracture through the use of machine learning techniques. He was incorporated to UPF with a Beatriu de Pinós (Marie Curie COFUND) grant in 2014, where he is manager of the Motion Capture laboratory and he is exploring the influence of gait and posture over musculoskeletal diseases. 